Marius V. Ionescu, Kasso A. Okoudjou*, and Luke G. Rogers

\title{
The strong maximum principle for Schrödinger operators on fractals
}

https://doi.org/10.1515/dema-2019-0034

Received February 14, 2019; accepted August 5, 2019

Abstract: We prove a strong maximum principle for Schrödinger operators defined on a class of postcritically finite fractal sets and their blowups without boundary. Our primary interest is in weaker regularity conditions than have previously appeared in the literature; in particular we permit both the fractal Laplacian and the potential to be Radon measures on the fractal. As a consequence of our results, we establish a Harnack inequality for solutions of these operators.

Keywords: analysis on fractals, Harnack’s inequality, maximum principles Sierpiński gasket, Schrödinger operators

MSC: Primary 35J15, 28A80; Secondary 35J25

\section{Introduction}

The goal of this note is to prove a strong maximum principle and related results for Schrödinger operators $L=$ $\Delta-v$ where $\Delta$ is a fractal Laplacian (to be defined below) and the potential $v$ is a non-negative measure on the fractal. When the fractal $K$ is the standard Sierpinski gasket or its unbounded analogue, Strichartz established strong maximum principles for solutions of the nonlinear equation $\Delta u=F(x, u)$ where $F: K \times \mathbb{R} \rightarrow \mathbb{R}$ is continuous and nonnegative in the sense that if $u(x) \geq 0$ then $F(x, u(x)) \geq 0$, see [1]. We consider algebraically simpler operators because our interest is in weakening the regularity conditions on both the Laplacian $\Delta$ and the potential, both of which we will permit to be measures.

In Section 2 we recall some basic facts about analysis on fractals and fractal blowups, details of which may be found in $[2,3]$. Section 3 contains the proof of the maximum principle and some comments on a Hopf-type lemma, and Section 4 has the proof of a Harnack inequality.

\section{Preliminaries}

We consider a connected self-similar set $K$ generated by an iterated function system $\left\{F_{1}, \ldots, F_{N}\right\}$ consisting of contractive maps on a complete metric space. To a finite word $\omega=\omega_{1} \omega_{2} \cdots \omega_{n} \in\{1, \ldots, N\}^{n}$ we associate $F_{\omega}=F_{\omega_{1}} \circ \cdots \circ F_{\omega_{n}}$ and a cell $C_{\omega}=F_{\omega}(K)$. We assume $K$ is post-critically finite, whence there is a finite set $V_{0}$ so that for any words $\omega, \omega^{\prime}$ we have $C_{\omega} \cap C_{\omega^{\prime}} \subset F_{\omega}\left(V_{0}\right) \cap F_{\omega^{\prime}}\left(V_{0}\right)$. From the set $V_{0}$, we inductively define $V_{n+1}=\cup_{i=1}^{N} F_{i}\left(V_{n}\right)$ and thereby obtain a countable dense subset $V_{\star}=\cup_{n} V_{n}$ of $K$. The topology of $K$ is

Marius V. Ionescu: Department of Mathematics, United States Naval Academy, Annapolis, MD, 21402-5002, USA;

E-mail: ionescu@usna.edu

*Corresponding Author: Kasso A. Okoudjou: Department of Mathematics and Norbert Wiener Center, University of Maryland, College Park, MD 20742, USA; E-mail: kasso@math.umd.edu

Luke G. Rogers: Department of Mathematics, University of Connecticut, Storrs, CT 06269-1009, USA;

E-mail:rogers@math.uconn.edu

Әopen Access. (c) BY (c) 2019 Marius V. Ionescu et al., published by De Gruyter. This work is licensed under the Creative Commons Attribution alone 4.0 License. 
generated by cells in the sense that any $x \notin V \star$ has a neighborhood base consisting of interiors of cells, while any $x \in V_{n}$ has a neighborhood base in which each set is a finite union of interiors of cells adjoined at $x$. We let $\mu$ denote the standard self-similar measure on $K$.

Following Kigami [2] we make the strong assumption that there is a resistance form $\mathcal{E}$, also called the energy, with domain dom $\mathcal{E} \subseteq L^{2}(K, \mu)$ that is obtained from a regular self-similar harmonic structure. This means that there is an irreducible, non-negative, symmetric, quadratic form $\varepsilon_{0}$ defined on the (finitedimensional) vector space of functions on $V_{0}$, and factors $0<r_{i}<1$ for each $i=1, \ldots, N$, such that setting $r_{\omega}=\prod_{1}^{n} r_{\omega_{j}}$ we have for $u \in \operatorname{dom} \mathcal{E}$

$$
\mathcal{E}_{n}(u, u):=\sum_{\omega \in\{1, \ldots, N\}^{n}} r_{\omega}^{-1} \varepsilon_{0}\left(u \circ F_{\omega}, u \circ F_{\omega}\right)
$$

and $\mathcal{E}(u, u)=\lim _{n} \mathcal{E}_{n}(u, u)$, where the latter sequence is non-decreasing (see Sections 2.3 and 2.4 of [2]). Those functions on which $\mathcal{E}_{n}$ is constant for $n \geq m$ are called piecewise harmonic at scale $m$. It follows that the pair $(\varepsilon$, dom $\varepsilon$ ) has the following properties: 1) $\mathcal{E}$ is a non-negative symmetric quadratic form on the linear space $\operatorname{dom} \varepsilon$, 2) $\varepsilon$ vanishes exactly on the constants and dom $\varepsilon$ modulo constants is a Hilbert space under $\varepsilon$, 3) any function on a finite subset of $K$ has an extension in $\operatorname{dom} \mathcal{E}$, 4) for any $p, q \in K$ the quantity

$$
R(p, q)=\sup \left\{|u(p)-u(q)|^{2} / \mathcal{E}(u, u): u \in \operatorname{dom} \mathcal{E} \text { and } \mathcal{E}(u, u)>0\right\}
$$

is finite, and 5) if $u \in \operatorname{dom} \mathcal{E}$ then so is $\bar{u}=\max \{0, \min \{u, 1\}\}$ and $\mathcal{E}(\bar{u}, \bar{u}) \leq \mathcal{E}(u, u)$. Moreover, $R(p, q)$ is a metric on $V_{\star}$ with completion homeomorphic to (and hence identified with) $K$, to which the continuous extension of $R$ is called the resistance metric; any function $u \in \operatorname{dom} \varepsilon$ satisfies

$$
|u(x)-u(y)|^{2} \leq \mathcal{E}(u, u) R(x, y) .
$$

It follows that any $u \in \operatorname{dom} \varepsilon$ is continuous with respect to the resistance metric; and $\varepsilon$ is a Dirchlet form on $L^{2}(K, \mu)$, see [4]. From the general theory of Dirichlet forms (see, for example, [5]) one then defines a nonpositive definite, self-adjoint (Dirichlet) Laplacian operator $\Delta$ for which $u \in \operatorname{dom} \Delta$ and $\Delta u=f \in C(K)$ by requiring

$$
\mathcal{E}(u, w)=-\int_{K} f w d \mu
$$

for all $w \in \operatorname{dom} \varepsilon$ such that $\left.w\right|_{V_{0}}=0$. If we only assume that $f \in L^{p}(K, \mu)$, then we say that $u \in \operatorname{dom}_{L^{p}} \Delta$. We will primarily work with an extension of the above definition in which $\Delta u$ is a finite signed Radon measure (see [6, Definition 4.1] and [7, Definition 6.7]): we say that $u \in \operatorname{dom}_{\mathcal{M}} \Delta$ and $\Delta_{\mathcal{M}} u=\sigma$ if $u \in \operatorname{dom} \mathcal{E}$ and

$$
\mathcal{E}(u, w)=-\int_{K} w d \sigma
$$

for all $w \in \operatorname{dom} \mathcal{E}$ such that $\left.w\right|_{V_{0}}=0$. Of course, we can view any function $f \in L^{1}(K, \mu)$ as the measure $f d \mu$. We assume that the Laplacian is self-similar in the sense that

$$
\Delta_{\mathcal{M}}\left(u \circ F_{\omega}\right)=r_{\omega} \mu_{\omega}\left(\Delta_{\mathcal{M}} u\right) \circ F_{\omega},
$$

for all $\omega \in\{1, \ldots, N\}^{n}$ and $n \geq 1$, where $\mu_{i}$ are the weights of the self-similar measure $\mu$ and $\mu_{\omega}=\prod_{j=1}^{n} \mu_{\omega_{j}}$ and $r_{\omega}$ is defined similarly (see the comments following Definition 4.2 of [6] why the definition of the localization of the measure valued Laplacian to a cell is not as straightforward as it may seem).

Our main results are also valid on bounded subsets of the fractal blowups considered by Strichartz [8]. An infinite blow-up without boundary points of the p.c.f. fractal $K$ is defined using a sequence $\alpha \in\{1, \ldots, N\}^{\mathbb{N}}$ such that $\alpha$ is not eventually constant. For $n \geq 1$ set $K_{n}=F_{\alpha_{1}}^{-1} F_{\alpha_{2}}^{-1} \ldots F_{\alpha_{n}}^{-1}(K)$. Then $\left\{K_{n}\right\}$ is an increasing sequence of sets and the infinite blow-up is defined to be $K_{\infty}=\bigcup_{n=1}^{\infty} K_{n}$. Both the energy $\mathcal{E}$ and the measure $\mu$ extend to $K_{\infty}$ in the obvious fashion, and we write $\varepsilon_{\infty}$ and $\mu_{\infty}$ for these extensions. The Laplacian $\Delta_{\infty}$ is defined weakly as before. 


\section{Maximum principle}

Let $v$ be a finite non-negative Radon measure. The Schrödinger operator $L$ with potential $v$ is defined on $\operatorname{dom}_{\mathcal{M}} \Delta$ by

$$
L u:=\Delta u-u v .
$$

The main result of this section is a maximum principle for the operator $L$. The proof goes via a result on subharmonic functions that relies on an argument of Kigami [4, Theorem 5.8(2)]; it has been used in various forms by other authors (for example, in [1, Theorem 2.1], [9, Lemma 4.3]). For the purposes of exposition we first prove a weak and then a strong maximum principle, though of course the former is a consequence of the latter.

Proposition 1. Let $u \in \operatorname{dom}_{\mathcal{M}} \Delta$ on $K$. If $C=C_{\omega}$ is a cell on which $\Delta u$ is a non-negative measure then $\max _{C} u \leq$ $\max _{\partial C} u$. Moreover, if $u$ attains its maximum at an interior point of $C$ then $u$ is constant on $C$.

Proof. Self-similarity of $\Delta$ ensures there is no loss of generality in taking $C=K$. For any $n$ and a point $p \in$ $V_{n} \backslash V_{0}$ there is a piecewise harmonic function at scale $n$ called $h_{p}$ that is harmonic on $K \backslash V_{n}$ with $h_{p}(p)=1$ and $h_{p}(q)=0$ if $q \in V_{n} \backslash\{p\}$. The maximum principle for harmonic functions ([2, Theorem 3.2.5]) implies $0 \leq h_{p} \leq 1$, and since $\left.h_{p}\right|_{V_{0}}=0$ we have

$$
0=\mathcal{E}\left(h_{p}, u\right)+\int_{K} h_{p} \Delta u \geq \mathcal{E}\left(h_{p}, u\right)=\mathcal{E}_{n}\left(h_{p}, u\right)=\sum_{q \sim_{n} p} c_{p q}(u(p)-u(q)),
$$

where the $c_{p q}>0$ are constants depending only on $\varepsilon_{n}$ and $q \sim_{n} p$ means that $q$ and $p$ are neighbors in $V_{n}$ (this is established in a similar manner as [2, Lemma 3.5.1]). Using this inductively beginning at $n=1$ and considering each point in $V_{n}$, we deduce that $u$ is bounded on $V_{\star}$ by $\max _{V_{0}} u$, whence the desired inequality follows by continuity of $u$.

Now suppose $u$ attains an interior maximum at $x$. We distinguish two cases according to whether $x \in V$ * or $x \notin V$ *.

If $x \in V_{\star}$ then it is in $V_{n}$ for some $n$. Let $q \sim_{n} x$ and from (3.2) and $u(x) \geq u(q)$ deduce both that $u(q)=u(x)$ and that $\int h_{x} \Delta u=0$. Since $h_{x}>0$ on the interiors of the $n$-cells containing $x$, we conclude that $\Delta u$ has no mass on these cells and thus that $u$ is harmonic on them. As $u$ is harmonic and all its boundary values on these cells equal $u(x)$ it is a constant function, and hence $u \equiv u(x)$ on a neighborhood of $x$.

If $x \notin V \star$ we fix an $n$ and the $n$-cell $C_{n}$ containing $x$. Let $h$ be harmonic on $C_{n}$ with $h=u$ on the boundary $\partial C_{n}$. We use another result of Kigami [2, Proposition 3.5.5 and Theorem 3.5.7] and [7, Theorem 6.8], namely that there is a non-negative Green kernel $g$ that inverts $-\Delta$ on $C_{n}$ with Dirichlet boundary data. Thus, for $y \in C_{n}$,

$$
u(y)=h(y)+\int_{K} g(y, z)(-\Delta u(d z)) \leq h(y),
$$

which simply says $u$ is subharmonic. However we then have

$$
u(x) \leq h(x) \leq \max _{\partial C_{n}} h(y)=\max _{\partial C_{n}} u(y) \leq u(x)
$$

where the first inequality is (3.3), the second is the maximum principle for harmonic functions, the equality is $u=h$ on $\partial C_{n}$, and the final inequality is that $u(x)$ is the maximum of $u$. Since equality must hold throughout we conclude $u=u(x)$ on $\partial C_{n}$. However (3.3) must also be an equality, and since $g(y, z)>0$ unless $z \in \partial C$ we find that $\Delta u$ has no mass on the interior of $C$ whence $u$ is harmonic and therefore constant. Again we have found $u \equiv u(x)$ on a neighborhood of $x$.

We conclude by noting $\{y: u(y)=u(x)\}$ is closed by continuity of $u$, open by the preceding reasoning, and contains $x$, so by connectivity it is $K$.

The preceding argument extends readily to our class of Schrödinger operators. For $u$ a function on $K$ or $K_{\infty}$ let $u^{+}(x)=\max \{u(x), 0\}$. 
Theorem 2 (Maximum principle for Schrödinger operators). Let $v$ be a non-negative Radon measure on $K_{\infty}$. Suppose $E \subset K_{\infty}$ is open and bounded, and consider $u \in \operatorname{dom}_{\mathcal{M}} \Delta_{\infty}$. If $L u=\Delta u-v u$ is a non-negative measure on $E$ then

$$
\max _{x \in E} u(x) \leq \max _{p \in \partial E} u^{+}(p) .
$$

Moreover, if $u$ achieves a positive maximum at an interior point $x \in E$ then $u$ is constant on the connected component of E containing $x$.

Proof. Observe that the asserted inequality is trivial if $u \leq 0$. Accordingly we may assume $U:=\{x \in E$ : $u(x)>0\} \neq \emptyset$. Then $\Delta u$ is a non-negative measure on $U$, so Proposition 1 is applicable to each cell contained in $U$. Moreover the proof of Proposition 1 implies there cannot be a strict maximum at $p \in V_{\star}$ if there is a scale $n$ such that all neighbors $q \sim_{n} p$ are in $U$. Thus the maximum of $u$ cannot only occur at an interior point to $U$ because every such point has a neighborhood in $U$ that is a cell or finite union of cells at a single scale. Since $u=0$ at any point $p \in \partial U$ that is interior to $E$, the maximum must be achieved on $\partial U \cap \partial E$, which implies the stated inequality.

If a positive maximum occurs at an interior point $x$ of $E$ it must also be that $x \in U$, which is open. If $x \notin V \star$ it is contained in the interior of a cell contained in $U$ and from Proposition $1 u$ is constant on this cell. If $x \in V_{\star}$ then there is neighborhood of $x$ in $U$ consisting of $x$ and the interiors of some cells meeting at $x$ and lying in $U$, but in this case the reasoning in the proof of Proposition 1 implies $u$ is constant on these cells. In summary, $u=u(x)$ on a neighborhood of $x$. However this implies the set $Y=\{y: u(y)=u(x)\}$ is open in $E$ because every such $y$ is necessarily in the open set $U$, and $Y$ is obviously closed because $u$ is continuous, so $u$ is constant on the connected component of $E$ containing $x$.

In the classical setting of a Euclidean space, one standard approach to obtaining the strong maximum principle from the weak maximum principle is to use the Hopf lemma. It is perhaps interesting to note that in the fractal setting we can prove a Hopf-type lemma at points in $V \star$ but have no corresponding results at points of $K \backslash V \star$ and therefore cannot use this approach to obtain a strong maximum principle.

To state our Hopf-type lemma we recall that the normal derivative [2, Definition 3.7.6] of a function at a point $p \in V_{0}$ may be written using the scale $m$ piecewise harmonic function $h_{p}^{(m)}$ which is 1 at $p$ and zero on $V_{m} \backslash\{p\}$ as

$$
\partial_{n} u(p)=\lim _{m \rightarrow \infty} \mathcal{E}\left(h_{p}^{(m)}, u\right)
$$

Lemma 3. Let $v$ be a non-negative Radon measure on $K$ and suppose $u \in \operatorname{dom}_{\mathcal{M}} \Delta$ satisfies $L u=\Delta u-u v$ is also non-negative measure. If there is $p \in V_{0}$ such that $u(p)>u(x)$ for all $x \in K \backslash\{p\}$ and also $u(p)>0$ then $\partial_{n} u(p)>0$.

Proof. If $n<m$ the difference $k^{(n, m)}=h_{p}^{(n)}-h_{p}^{(m)}$ is zero at $p$ and the points $q \sim_{n} p$, and is equal to $h_{p}^{(n)}(q)>0$ at each $q \sim_{m} p$; it is otherwise harmonic, so the minimum principle for harmonic functions implies it is nonnegative. Now $u(p)>0$ and $u$ is continuous so there is $n$ such that $u$ is positive on the support of $k^{(n, m)}$. For this $n$ and any $m>n$ we see $\Delta u \geq u v$ is a non-negative measure on the support of $k^{(n, m)}$ and therefore

$$
\mathcal{E}\left(h_{p}^{(n)}-h_{p}^{(m)}, u\right)=-\int\left(h_{p}^{(n)}-h_{p}^{(m)}\right) \Delta u \leq 0
$$

which gives $\partial_{n} u(p)=\lim _{m \rightarrow \infty} \mathcal{E}\left(h_{p}^{(m)}, u\right) \geq \varepsilon\left(h_{p}^{(n)}, u\right)=\sum_{q \sim_{n} p} c_{p q}(u(p)-u(q))$ for some values $c_{p q}>0$ depending on $\varepsilon_{n}$. The fact that $u(p)>u(q)$ for all $q$ concludes the proof.

\section{Harnack inequality}

In the classical setting the strong maximum principle for $L u \geq 0$ implies a Harnack inequality for solutions of $L u=0$, see [10, Section 8.8]. We show that this is the case in our setting. 
Before stating the Harnack inequality, we note that the equation $L u=0$ for $L$ as in (3.1) has solutions for sufficiently small measures $v$ on $K$, because with boundary data a harmonic function $h$, the operator defined using the continuous non-negative Green kernel $g(x, y)$ by

$$
u \mapsto h+\int g(x, y) u(y) v(d y)
$$

is contracting in the uniform norm provided $\int g(x, y) v(d y)<1$. Moreover, on the cell $F_{\omega}(K)$ the Green kernel is $r_{\omega} g\left(F_{\omega}^{-1}(x), F_{\omega}^{-1}(y)\right)$ where $r_{\omega}=\prod_{j=1}^{|\omega|} r_{\omega_{j}}$ by [2, Proposition 3.5.5]. It follows for any $v$ that we can take $|\omega|$ sufficiently large so that contractivity of the analogous operator on $F_{\omega}(K)$ is valid, ensuring local solutions exist everywhere. This argument extends to the case when one has a finite union of cells.

Theorem 4 (Harnack inequality). On $K$, fix a non-negative finite Radon measure $v$. Suppose $u \in \operatorname{dom}_{\mathcal{M}}(\Delta)$ satisfies both $u \geq 0$ and $L u=0$, where $L$ is as in (3.1) and the latter is an equality of measures. If $E$ is a compact subset of a connected component of $K \backslash V_{0}$ then there is a constant $A$ that depends only on $E$ such that $\max _{E} u \leq$ $A \min _{E} u$.

The proof makes use of the following result, which may be of independent interest.

Proposition 5. For $v$ a non-negative finite Radon measure on $\mathrm{K}$ let

$$
\mathcal{A}=\left\{u \in \operatorname{dom}_{\mathcal{M}}(\Delta): L u=0 \text { as measures and } 0 \leq u \leq 1\right\} .
$$

Then $\mathcal{A}$ is compact in the uniform norm.

Proof. For any $u \in \mathcal{A}$ with $\left.u\right|_{V_{0}}=0$ we have

$$
\mathcal{E}(u, u)=-\int u \Delta u=-\int u^{2} d v
$$

so that $|u| \leq 1$ implies $|\mathcal{E}(u, u)| \leq v(K)=M<\infty$. Applying the estimate (2.2) we have

$$
|u(x)-u(y)|^{2} \leq \mathcal{E}(u, u) R(x, y) \leq M R(x, y) .
$$

If instead, $\left.u\right|_{V_{0}} \neq 0$, then choose a harmonic function $h$ so $\left.(u-h)\right|_{V_{0}}=0$ and since $|h| \leq 1$ on $V_{0}$ we have $|h(x)-h(y)|^{2} \leq 2 R(x, y)$, so (4.1) holds with $M$ replaced by $M+2$. However an estimate of the type (4.1) implies $\mathcal{A}$ is equicontinuous, and since the definition of $\mathcal{A}$ implies it is equibounded, an application of the Arzela-Ascoli theorem then yields that it is precompact in the uniform norm.

Now suppose $\left\{u_{n}\right\} \subset \mathcal{A}$ and $u_{n} \rightarrow u$ uniformly. If $w \in \operatorname{dom}(\mathcal{E})$ and $\left.w\right|_{V_{0}}=0$ we know $w$ is continuous and therefore bounded so Hölder's inequality provides

$$
\left|\mathcal{E}\left(u_{n}-u_{m}, w\right)\right|=\left|\int_{K} w \Delta\left(u_{n}-u_{m}\right)\right|=\left|\int_{K} w\left(u_{n}-u_{m}\right) d v\right| \leq M\left\|u_{n}-u_{m}\right\|_{\infty},
$$

and therefore $u_{n}$ is Cauchy in $\operatorname{dom}(\varepsilon)$. However $\operatorname{dom}(\varepsilon)$ is a Hilbert space under the norm $\varepsilon+\|\cdot\|_{\infty}$, so we conclude that $u \in \operatorname{dom}(\mathcal{E})$ and $\mathcal{E}(u, w)=-\int w u d v$, whence $\Delta u=u v$. This implies $u \in \mathcal{A}$, so $\mathcal{A}$ is closed, and in light of our Arzela-Ascoli argument, compact in the uniform norm.

Proof of Theorem 4. Let

$$
\mathcal{A}=\left\{u \in \operatorname{dom}(\Delta): L u=0,0 \leq u \leq 1 \text { and } \max _{V_{0}} u=1\right\} .
$$

Evidently this is a closed subset of the space of functions considered in Proposition 5 and is therefore compact in the uniform norm. Hence the quantity $a=\inf _{\mathcal{A}} \min _{x \in E} u(x)$ is achieved by some $\tilde{u} \in \mathcal{A}$. Either $a=1$ or $\tilde{u}$ is non-constant and the hypothesis $\tilde{u} \geq 0$ implies $a>0$ by the strong minimum principle.

Now take $u$ as in the statement of the theorem. It is continuous and the strong maximum principle is applicable, so it achieves its maximum on $V_{0}$. Accordingly, $u /\left(\max _{V_{0}} u\right) \in \mathcal{A}$ and has minimum at least $a$ on $E$. The result follows with $A=1 / a$. 
Remark 6. The above immediately implies the corresponding result in the setting of a bounded subset of $K_{\infty}$ because such a set is contained in a (sufficiently large) copy of $K$, and we can transfer the theorem directly to this setting using the self-similarity of the energy.

Acknowledgments: We thank the reviewers for their insightful comments. K. A. O. was partially supported by a grant from the Simons Foundation \#319197, the U. S. Army Research Office grant W911NF1610008, the National Science Foundation grant DMS 1814253, and an MLK visiting professorship. L. G. R. was partially supported by the National Science Foundation grant DMS 1659643.

\section{References}

[1] Strichartz R. S., Some properties of Laplacians on fractals, J. Funct. Anal., 1999, 164(2), 181-208

[2] Kigami K., Analysis on fractals, Cambridge Tracts in Mathematics, vol. 143, Cambridge University Press, Cambridge, 2001

[3] Strichartz R. S., Differential equations on fractals, A tutorial, Princeton University Press, Princeton, N], 2006

[4] Kigami K., Harmonic calculus on p.c.f. self-similar sets, Trans. Amer. Math. Soc., 1993, 335(2), 721-755

[5] Fukushima M., Oshima Y., Takeda M., Dirichlet forms and symmetric Markov processes, extended ed., de Gruyter Studies in Mathematics, vol. 19, Walter de Gruyter \& Co., Berlin, 2011

[6] Strichartz R. S., Usher M., Splines on fractals, Math. Proc. Cambridge Philos. Soc., 2000, 129(2), 331-360

[7] Kigami K., Harmonic analysis for resistance forms, J. Funct. Anal., 2003, 204(2), 399-444

[8] Strichartz R. S., Fractals in the large, Canad. J. Math., 1998, 50(3), 638-657

[9] Rogers L. G., Estimates for the resolvent kernel of the Laplacian on p.c.f. self-similar fractals and blowups, Trans. Amer. Math. Soc., 2012, 364(3), 1633-1685

[10] Gilbarg D., Trudinger N. S., Elliptic partial differential equations of second order, Classics in Mathematics, Springer-Verlag, Berlin, 2001, Reprint of the 1998 edition 